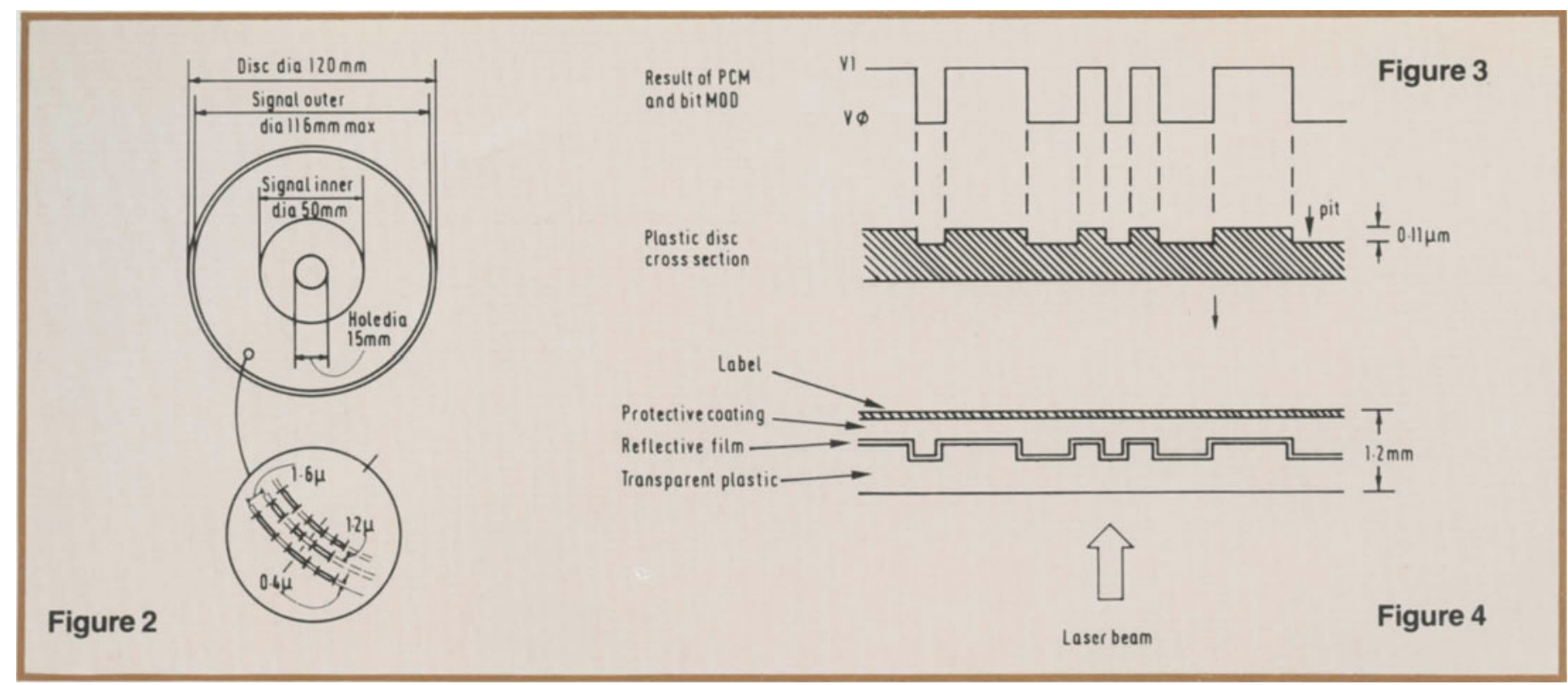

\section{Disc Structure}

During the disc mastering process a series of 'pits' are pressed into the surface of a transparent plastic disc. The pit pattern corresponds to the PCM (Pulse Code Modulation) audio signal.

The depth of each pit is $0.11 \mu \mathrm{m}$. To give an idea of the depth involved, the paper on which this text is printed is approximately $45 \mu \mathrm{m}$ thick (approximately 400 times thicker).

The pits form a spiral track that starts near the centre of the disc and spirals outward. Each disc is capable of storing a maximum of 75 minutes of information.
The pit width is $0.4 \mu \mathrm{m}$ and the spacing between adjacent tracks is $1.2 \mu \mathrm{m}$. This gives a pitch of $1.6 \mu \mathrm{m}$.

The pitted surface of the disc is coated with a reflective material. During playback the disc is scanned from underneath by a laser beam (see figure 4) which is reflected back to photo-diode sensors.

The pits appear as 'bumps' which modulate the scanning laser beam and produce a modulated output from the sensors.
The recovered signal corresponds precisely with the original PCM audio signal used during mastering.

A coating of clear plastic is applied to protect the pits and reflective coating from mechnical damage, and the disc label is attached to the upper surface. 